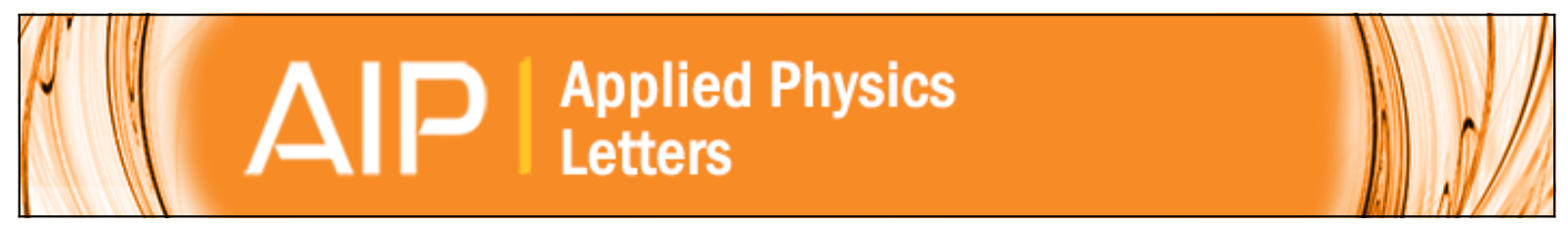

\title{
High Q -factor colloidal nanocrystal-based vertical microcavity by hot embossing technology
}

Luigi Martiradonna, Luigi Carbone, Milena De Giorgi, Liberato Manna, Giuseppe Gigli, Roberto Cingolani, and Massimo De Vittorio

Citation: Applied Physics Letters 88, 181108 (2006); doi: 10.1063/1.2200748

View online: http://dx.doi.org/10.1063/1.2200748

View Table of Contents: http://scitation.aip.org/content/aip/journal/apl/88/18?ver=pdfcov

Published by the AIP Publishing

\section{Articles you may be interested in}

Optical properties of II-VI colloidal quantum dot doped porous silicon microcavities

Appl. Phys. Lett. 96, 161106 (2010); 10.1063/1.3404183

Enhanced photoluminescence from embedded $\mathrm{PbSe}$ colloidal quantum dots in silicon-based random photonic crystal microcavities

Appl. Phys. Lett. 92, 261110 (2008); 10.1063/1.2954007

Fabrication and characterization of two-dimensional photonic crystal microcavities in nanocrystalline diamond Appl. Phys. Lett. 91, 201112 (2007); 10.1063/1.2813023

Impact of disorder on high quality factor III-V nitride microcavities

Appl. Phys. Lett. 89, 261101 (2006); 10.1063/1.2420788

Experimental demonstration of high quality factor, $\mathrm{x}$-dipole modes in In As/In P quantum dot photonic crystal microcavity membranes

Appl. Phys. Lett. 89, 091115 (2006); 10.1063/1.2338662

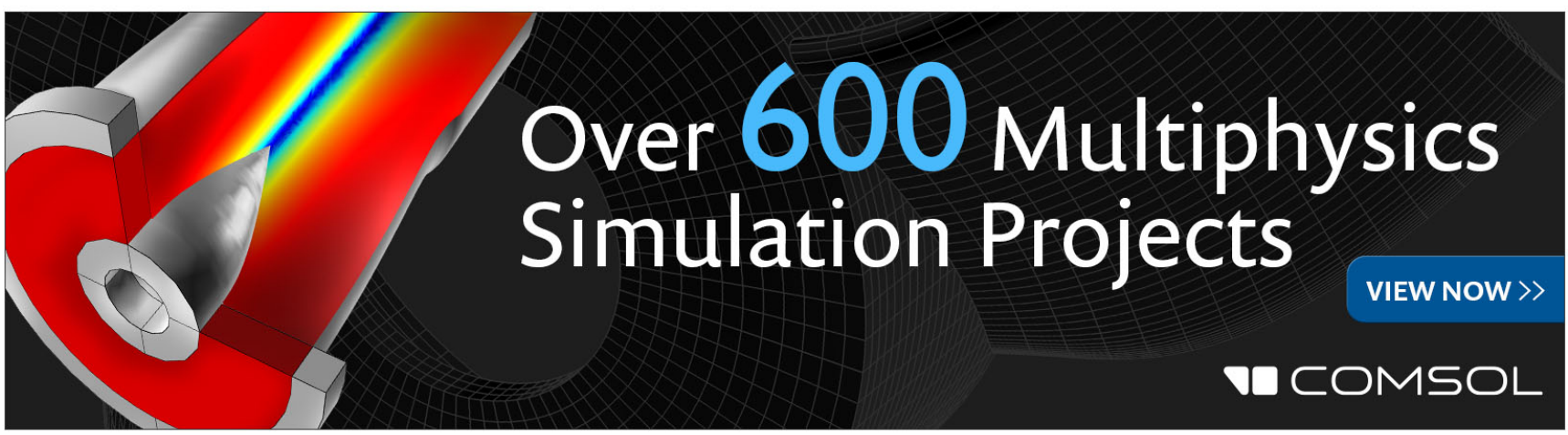




\title{
High Q-factor colloidal nanocrystal-based vertical microcavity by hot embossing technology
}

\author{
Luigi Martiradonna, ${ }^{\text {a) }}$ Luigi Carbone, Milena De Giorgi, Liberato Manna, Giuseppe Gigli, \\ Roberto Cingolani, and Massimo De Vittorio \\ National Nanotechnology Laboratory, CNR-INFM, Technological District-ISUFI, University of Lecce, \\ Via per Arnesano, Km 5, I-73100 Lecce, Italy
}

(Received 10 November 2005; accepted 18 March 2006; published online 3 May 2006)

\begin{abstract}
We report on the fabrication and optical characterization of vertical hybrid microcavities in which a layer of colloidal nanocrystals dispersed in an organic matrix is embedded between two inorganic mirrors. The devices are fabricated by a technique based on the unconventional use of the hot embossing technology, which allows a very fine control of the cavity length. The technique exploits a $\lambda$-thick microstructured dielectric top mirror pressed onto the bottom one, previously coated with the active layer, to sandwich the cavity and precisely control its thickness. Room-temperature photoluminescence measurements show a $Q$ factor as high as 146 for our devices. (C) 2006 American Institute of Physics. [DOI: 10.1063/1.2200748]
\end{abstract}

Colloidal nanocrystals (NCs) are an emerging class of functional materials for optoelectronics, electronic, and diagnostic applications. They are chemically synthesized in solution, at low cost, with high throughput ${ }^{1,2}$ and with a full control of their size and shape. The emission wavelength can be finely tuned in the whole visible spectral range by controlling the size of the clusters. Additionally, the fluorescence efficiency and, in particular, the optical stability of the nanocrystals can be greatly improved by modifying the particle surface in a core/shell configuration. ${ }^{3}$

Several proposed applications of nanocrystals are in the area of optical devices. Recent developments in the fabrication of organic light emitting diodes ${ }^{4,5}$ (OLEDs) exploited blends of nanocrystals and organic compounds, merging the typical easy functionalization and low-cost processing of organic materials with the high chemical stability of traditional inorganic semiconductors. These characteristics can be jointly exploited in order to develop innovative functional devices. $^{6-9}$

Because of the inhomogeneous broadening, the emission spectrum of an ensemble of NCs shows a typical peak with a full width at half maximum (FWHM) of about $100 \mathrm{meV}$. In order to improve the spectral purity and directionality of NCbased emitting devices, the active material can be confined into optical microcavities. The optical and electrical properties of nanoparticles confined in nanocavities of high Purcell factor ${ }^{10}$ are of great interest for the fabrication of enhanced efficiency resonant cavity LEDs and extremely low-threshold hybrid lasers. Various works have reported on the fabrication of hybrid organic-inorganic vertical microcavities ${ }^{11-13}$ realized by spin coating a thin layer of an organic/NC blend onto an inorganic distributed Bragg reflector (DBR). The top mirror is typically a metallic layer deposited by means of thermal evaporation or a second DBR deposited by either sputtering or evaporation techniques. However, metallic mirrors show typical reflectivities lower than $90 \%$ with a strong absorption in the visible spectral range, whereas the direct evaporation of high optical quality dielectric layers on the active layer induces high thermal

${ }^{a)}$ Electronic mail: luigi.martiradonna@unile.it stress for long times on the $\mathrm{NC} /$ polymer blend. In order to avoid these problems a different approach has been proposed, which consists of spin coating an organic film onto a high-reflectivity DBR, on which a second DBR is mechanically pressed. ${ }^{14}$ This simple technique does not affect the properties of the active layer but, as a drawback, it allows a poor control of the distance between the two mirrors, which determines the resonance wavelength of the structure, due to the softness of the active material.

In this letter we report on the fabrication of high $Q$-factor vertical microcavities embedding $\mathrm{CdSe} / \mathrm{ZnS}$ core/ shell colloidal nanocrystals. These hybrid vertical microcavities have been obtained through an unconventional use of the hot embossing (HE) technique, which is a powerful method to replicate the pattern of a hard master on a soft thermoplastic layer. ${ }^{15}$ This assembling approach allowed us to improve the control on the resonance wavelength of the microcavity and, at the same time, to decrease the thermal stress of the active layer.

A schematic illustration of the fabrication process of the $\lambda$-thick hybrid vertical microcavity is displayed in Fig. 1. Two DBRs were deposited by electron-beam evaporation of alternating quarter wavelength layers of $\mathrm{TiO}_{2}$ and $\mathrm{SiO}_{2}$ on both $\mathrm{Si}$ and quartz substrates with reflectivities of $99 \%$ and $98.3 \%$, respectively.

Nearly spherical, core/shell CdSe/ZnS nanocrystals were prepared by using standard methods, ${ }^{16,17}$ showing emission at $580 \mathrm{~nm}(\mathrm{FWHM}=40 \mathrm{~nm})$ and high absorption in the ultraviolet spectral range (extinction coefficient ${ }^{18} \varepsilon$ typically greater than $\left.10^{5} \mathrm{~cm}^{-1} \mathrm{M}^{-1}\right)$. The NCs were dispersed ${ }^{19}$ in a matrix of poly(methylmethacrylate) (PMMA) diluted in chlorobenzene $\left(\mathrm{C}_{6} \mathrm{H}_{5} \mathrm{Cl}\right)$. A thin film of such blend was spin coated on the lower DBR, with a thickness roughly corresponding to the cavity length $L_{C}$.

The hard master was realized on the second DBR, deposited on a silicon substrate, by the direct lithographic patterning of a layer of SU8-2000 negative resist whose glasstransition temperature $T_{G}$ becomes higher than $200{ }^{\circ} \mathrm{C}$ after a curing process. The pattern consisted of a sequence of $25 \mu \mathrm{m}$ wide parallel stripes, with a period of $250 \mu \mathrm{m}$. This hard microstructured mold was used for the control of the 


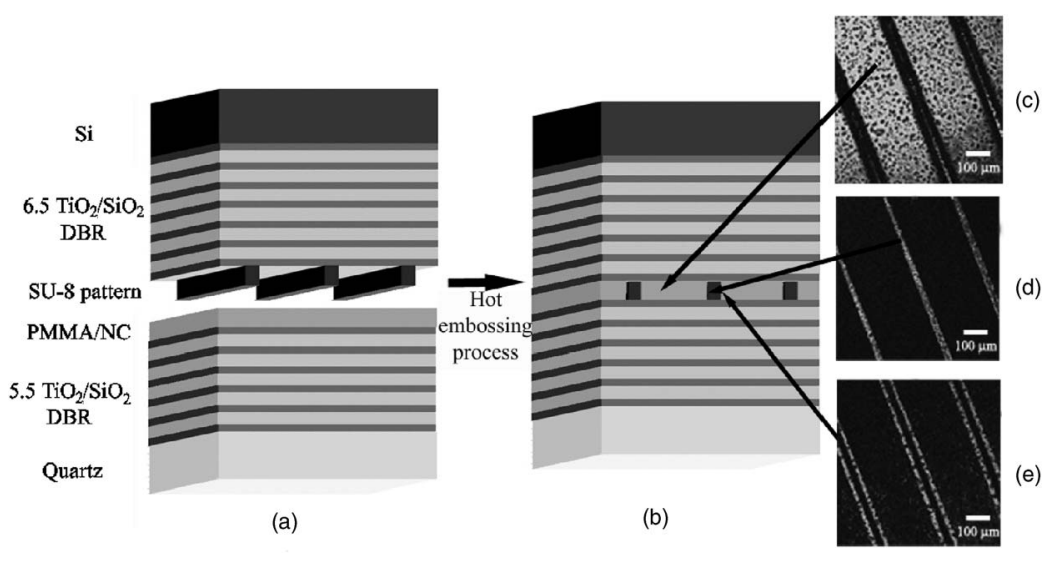

FIG. 1. (a) Scheme of the two dielectric DBRs covered by a patterned and cured SU8-2000 resist and by a thin film of the PMMA/NC blend, respectively. (b) The complete sandwiched vertical cavity structure obtained by hot embossing technique. (c)-(e) Spatially resolved PL analysis of the hybrid microcavity by means of a confocal system. Three different resonating regions have been clearly evidenced in the $\mu$-PL maps recorded from a $680 \times 680 \mu \mathrm{m}^{2}$ area of the microcavity: the regions, in the recesses of the master (c), under the SU-8 pattern (d), and near the pattern (e), have been detected at 616,590 , and $650 \mathrm{~nm}$, respectively. resonance wavelength of the device, the patterned structure acting as a physical spacer between the two dielectric mirrors. The hard master is not shrunken during the mechanical assembling, therefore, its final thickness is univocally determined by the SU-8 spin-coating parameters, with an overall uncertainty typically lower than $5 \mathrm{~nm}$. The thickness tuning and the calibration of the HE process parameters lead to a fine control of the cavity length and of the resonance wavelength. Moreover, the accomplishment of negative sidewalls in the SU8-2000 pattern results in a strong adhesion and bonding between the two parts.

The final assembling of the cavity has been realized by means of a $\mathrm{HE}$ press heated at $180{ }^{\circ} \mathrm{C}$.

In order to obtain a perfect match between the resonance wavelength and the emission wavelength of the active material, the mold thickness has been chosen by taking into account the expected presence of a residual bottom layer of soft material under the SU-8 pattern, typically not lower than $10 \mathrm{~nm}$, acting as a further spacer between the DBRs. The thickness of this bottom layer is strictly dependent on the hot embossing conditions, therefore, both the mold thickness and the HE process parameters have been varied in order to obtain the right resonance conditions along the device.

A critical factor which must be taken into account to have a good spatial uniformity of the resonance wavelength is the quality of the imprint tool, which has to guarantee a perfect uniformity of the applied pressure and a controlled parallelism between the plates. An angle of misalignment between the plates, in fact, leads to an in plane variation of the physical distance between the two mirrors and to a shift of the resonance wavelength. The mechanical press used in this work was affected by a slight misalignment angle of 12 in.; good spatial uniformity of the cavity length has thus been obtained in about $200 \times 200 \mu \mathrm{m}^{2}$ wide regions of the samples.

In order to check the spatial distribution of the microcavity and, therefore, to optimize the assembling parameters, room-temperature photoluminescence (PL) maps have been collected on our samples by means of an Olympus FluoView 1000 confocal laser scanning microscope, having a spatial resolution better than $1 \mu \mathrm{m}$. The detection wavelength has been varied from 540 to $680 \mathrm{~nm}$, corresponding to the emission spectral range of the NCs, with a spectral resolution of $2 \mathrm{~nm}$. The correlation between the emission wavelength detected by the $\mu$-PL maps, the resonance wavelength, and the cavity length is straightforward and allows us to determine the local thickness of the soft active material inside the microcavity, without disassembling the device.
Figures 1(c)-1(e) show the $\mu$-PL maps recorded on a $680 \times 680 \mu \mathrm{m}^{2}$ area of a microcavity used for the calibration of the assembling process. As it can be inferred from these images, three different resonating regions can be identified. The $25 \mu \mathrm{m}$ wide stripes with a bright photoluminescence in Fig. 1(d) correspond to the SU-8 spacers. By virtue of its transparency and higher refraction index $(n=1.69)$ as compared to the PMMA/NC blend $(n=1.55)$, the SU-8 pattern conveys a fraction of the NCs emission and behaves itself as a resonant cavity between the DBRs. In Fig. 1(c) the detection wavelength corresponds to the emission peak of the large recessed regions filled by the active material. The dark spots correspond to nonemitting zones of optically inert PMMA molecules in the active layer. These regions are probably formed because of phase separation induced by repulsive interactions between the polymer chains and the surfactants that coat the nanocrystals surface. Finally, the borders of the cavity near the SU-8 stripes show a different resonant wavelength [Fig. 1(e)], thus revealing a different cavity optical length as compared to the recessed region. This variation of the distribution of the thermoplastic material has been interpreted according to the filling mechanisms described by Heyderman et al. ${ }^{15}$ in the hot embossing processes. During the assembling phase, the raised pattern penetrates into the soft material, which fills the recessed regions of the mold. The NC/polymer blend moves laterally into the cavity, creating raised regions at the borders, while the central cavity region is initially not interested by this flow, so that a thin empty volume can remain trapped between the active layer and the upper DBR. This spatial variation of the blend thickness leads to local changes of the optical path covered by the resonating radiation. Longer assembling times, higher applied pressures, or a decrease of the cavity dimensions in the nanoscale range completely remove this thickness variation, improving the lateral uniformity.

The above optical investigation allowed us to carefully test and calibrate the imprinting process for the fabrication of the microcavities. Finally, in order to evaluate the performances of our devices, the peak linewidth and the $Q$ factor were detected by PL measurements by using a $\mathrm{He}-\mathrm{Cd}$ laser source as excitation source. The laser was focused on a $100 \mu \mathrm{m}$ wide spot on the sample by a (numerical aperture) $\mathrm{NA}=0.18$ quartz lens, which was used also to collect the emission in backscattering. Figures 2 compare the emission spectrum of the NCs inside the microcavity to the reflectivity spectra of the two DBRs (a) and to the broad emission of the nanocrystals outside the cavity (b). Due to a slight redshift of 


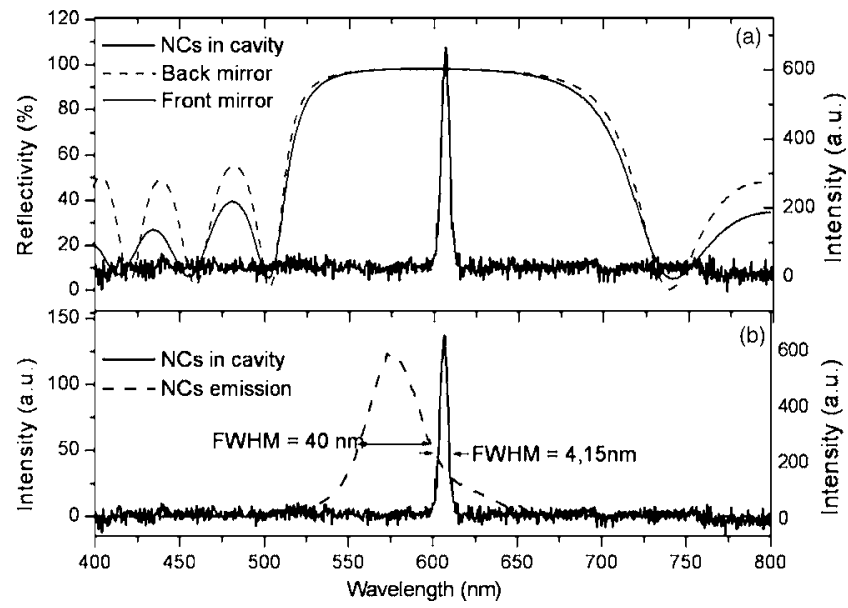

FIG. 2. PL analysis of the hybrid microcavity. (a) Comparison between the emission of NCs embedded in the microcavity (bold line) and the reflectivity spectra of the back (dashed line) and front (solid line) mirrors. (b) Comparison between the emission of NCs with (solid bold line) and without (dashed bold line) the presence of the microcavity.

the reflectivity spectra on the grown DBRs, the best measured cavity $Q$ factor has been obtained for a resonance wavelength higher than the NCs emission peak. The $Q$ factor is equal to 146 for the peak in Fig. 2, which is the highest reported in the literature for microcavities embedding colloidal nanocrystals. Moreover, the reflectivity spectrum of the microcavity was simulated by using the transfer matrix method, showing a theoretical $Q$ factor of 341. The discrepancy between the simulated and measured values was attributed to the high numerical aperture of the detection system. The resonance wavelength, in fact, undergoes a spectral shift depending on the chosen angle of detection; our PL setup detects the cavity emission through a NA=0.18 focus lens, therefore giving an emission spectrum integrated over an angular range from $0^{\circ}$ to $7^{\circ}$ and, consequently, overestimating the peak linewidth.

In summary, we have applied the hot embossing technology to the fabrication of photonic vertical microcavities embedding $\mathrm{CdSe} / \mathrm{ZnS}$ core/shell colloidal nanocrystals dispersed in a polymer matrix. As opposed to commonly fabricated organic vertical cavity devices, this method allows one to carefully control the active material waveguide layer through the control of the DBR/mold patterning depth and through the HE process. The effectiveness of this technology has been demonstrated by room-temperature photoluminescence (PL) spectra recorded on the fabricated microcavity, which show a sharp emission peak with a $Q$ factor as high as 146. In addition, this technique is intrinsically suitable for the realization of both vertical and in-plane optically confined structures. In fact, quasi-three-dimensional (3D) microcavities are possible by patterning the mold with a carefully designed two-dimensional photonic crystal geometry, in order to further confine the NCs emission in the substrate plane.

The authors gratefully thank Marco Anni and Pier Paolo Pompa for fruitful discussions. The authors also acknowledge the expert technical help of Paolo Cazzato, Angelo Melcarne, Gianmichele Epifani, and Stefania D'Amone. This work was partially supported by FAR 297 and FISRNanophotonics projects.

${ }^{1}$ A. Kasuya, R. Sivamohan, Y. A. Barnakov, I. M. Nirasawa, V. R. Romanyuk, V. Kumar, S. V. Mamykin, K. Tohji, B. Jeyadevan, K. Shinoda, T. Kuda, O. Terasaki, Z. Liu, R. V. Belosludov, V. Sundararajan, and Y. Kawazoe, Nat. Mater. 3, 99 (2004).

${ }^{2}$ A. P. Alivisatos, Science 271, 933 (1996).

${ }^{3}$ W. J. Parak, D. Gerion, T. Pellegrino, D. Zanchet, C. Micheel, S. C. Williams, R. Boudreau, M. A. Le Gros, C. A. Larabell, and A. P. Alivisatos, Nanotechnology 14, R15 (2003).

${ }^{4}$ J. R. Sheats, H. Antoniadis, M. Hueschen, W. Leonard, J. Miller, R. Moon, D. Roitman, and A. Stocking, Science 273, 884 (1996).

${ }^{5}$ Y. Q. Li, A. Rizzo, M. Mazzeo, L. Carbone, L. Manna, R. Cingolani, and G. Gigli, J. Appl. Phys. 97, 113501 (2005).

${ }^{6}$ K. A. Dick, K. Deppert, M. W. Larsson, T. Martensson, W. Seifert, L. R. Wallenberg, and L. Samuelson, Nat. Mater. 3, 380 (2004).

${ }^{7}$ N. Tessler, V. Medvedev, M. Kazes, S. H. Kan, and U. Banin, Science 295, 1506 (2002).

${ }^{8}$ M. Bruchez, M. Moronne, P. Gin, S. Weiss, and A. P. Alivisatos, Science 281, 2013 (1998).

${ }^{9}$ X. Y. Wu, H. J. Liu, J. Q. Liu, K. N. Haley, J. A. Treadway, J. P. Larson, N. F. Ge, F. Peale, and M. P. Bruchez, Nat. Biotechnol. 21, 41 (2003).

${ }^{10}$ E. M. Purcell, Phys. Rev. E 69, 681 (1949).

${ }^{11}$ J. Roither, W. Heiss, D. V. Talapin, N. Gaponik, and A. Eychmuller, Appl. Phys. Lett. 84, 2223 (2004).

${ }^{12}$ C. E. Finlayson, D. S. Ginger, and N. C. Greenham, Appl. Phys. Lett. 77, $2500(2000)$.

${ }^{13}$ C. B. Poitras, M. Lipson, H. Du, M. A. Hahn, and T. D. Krauss, Appl. Phys. Lett. 82, 4032 (2003).

${ }^{14}$ M. Theander, T. Granlund, D. M. Johanson, A. Ruseckas, V. Sundstrom, M. R. Andersson, and O. Inganas, Adv. Mater. (Weinheim, Ger.) 13, 323 (2001).

${ }^{15}$ L. J. Heyderman, H. Schift, C. David, J. Gobrecht, and T. Schweitzer, Microelectron. Eng. 54, 229 (2000).

${ }^{16}$ P. Reiss, J. Bleuse, and A. Pron, Nano Lett. 7, 781 (2002).

${ }^{17}$ B. O. Dabboussi, J. Rodriguez-Viejo, F. V. Mikulec, J. R. Heine, H. Mattoussi, R. Ober, K. F. Jensen, and M. G. Bawendi, J. Phys. Chem. B 101, 9463 (1997).

${ }^{18}$ W. W. Yu, L. Qu, W. Guo, and X. Peng, Chem. Mater. 15, 2854 (2003).

${ }^{19}$ The concentration of NCs in the PMMA blend was optimized in order to have low surface roughnesses and a high photoluminescence efficiency. The samples roughness was estimated by means of atomic force microscopy (AFM) measurements collected with an easy dynamic force microscope (E-DFM) by Nanosurf in dynamic mode. Photoluminescence efficiency measurements on NCs/PMMA thin films spin coated on quartz substrates have been carried out according to the method proposed by Greenham et al. [N. C. Greenham, I. D. W. Samuel, G. R. Hayes, R. T. Phillips, Y. A. R. R. Kessener, S. C. Moratti, A. B. Holmes, and R. H. Friend, Chem. Phys. Lett. 241, 89 (1995)]. Molar concentrations of $M_{\mathrm{PMMA}}=3.492 \times 10^{-5} \mathrm{~mol} / 1$ for the polymer and $M_{\mathrm{NCs}}=3.4451$ $\times 10^{-1} \mathrm{~mol} / 1$ for the nanocrystals in chlorobenzene have been finally chosen. 